\title{
X-ray polarization properties of the accretion column in magnetic CVs
}

\author{
G. Matt \\ Dipartimento di Fisica, Università degli Studi Roma Tre, via della Vasca Navale 84, 00146 Roma, Italy \\ e-mail: matt@fis.uniroma3.it \\ Received 10 November 2003 / Accepted 26 April 2004

\begin{abstract}
The accretion column in magnetic Cataclysmic Variables may have a non negligible Thomson optical depth. A fraction of the thermal radiation from the post-shock region may therefore be scattered - and then polarized - before escaping the column. Moreover, part of the thermal radiation is reflected - and again polarized - by the White Dwarf surface. We show that $\mathrm{X}$-ray polarimetry can provide valuable and probably unique information on the geometry and physical parameters of the accretion column by calculating, by means of Monte Carlo simulations, the expected polarization properties of magnetic CVs as a function of the geometrical parameters (assuming a cylindrical geometry) and the Thomson optical depth of the column. We find that degrees of polarization as high as about $4 \%$ can be present, and apply our calculations to the archetypal magnetic CV, AM Herculis.
\end{abstract}

Key words. polarization - X-rays: binaries - X-rays: individuals: AM Herculis

\section{Introduction}

The magnetic field in some subclasses of Cataclysmic Variables (notably in Polars and in at least a fraction of intermediate Polars; see Warner 1995 for a complete overview on CVs) is strong enough to channel the accreting matter along the field lines. For a dipolar field, this means that the accretion occurs via accreting columns on one or both the magnetic poles; the misalignement between the spin and magnetic axes results in pulsed emission.

Hard X-rays are then produced by optically thin thermal line and continuum emission in the so-called post-shock region, where temperatures can reach values as large as several tens of keV (e.g. Frank et al. 1992; Cropper et al. 1999). Thermal emission is expected to be non-polarized (see next section); however, the Thomson optical depth in the accretion column, while probably less than unity, may be not negligible. Indeed, Hellier et al. (1998) found significant broadening in the iron $\mathrm{K} \alpha$ line of several magnetic CVs, which they interpreted as due to Compton broadening. Thomson scattered radiation is polarized, provided that the geometry is not spherical. It is therefore to be expected that the hard X-ray emission in magnetic CVs is polarized, with the net polarization degree increasing with the Thomson optical depth of the accretion column.

In this paper we calculate, by means of Monte Carlo simulations, the polarization properties of the accretion column in magnetic CVs. In Sect. 2 the numerical code is described, while the results (including an application to the archetypal Polar,
AM Herculis) are presented in Sect. 3. Results are then summarized, and observational perspectives discussed, in Sect. 4.

\section{Calculations}

The Monte Carlo technique adopted in our code is described in Matt (1993) and Matt et al. (1989; 1996). A full Compton scattering matrix is adopted (McMaster 1961). However, in the energy range under consideration $(\$ 20 \mathrm{keV})$, the scattering matrix basically reduces to the Rayleigh one. We assumed that primary photons (i.e. photons before scattering) are unpolarized. Bremsstrahlung photons have the polarization, i.e. the electric, vector perpendicular to the interaction plane, and the random velocity field ensures that the radiation has a null net polarization. In practice, in the code each photon is assigned a random polarization vector. After being emitted, the photon path is followed until it leaves the accretion column. We assumed total ionization of the matter, i.e. no photoelectric absorption.

We assumed, for simplicity, a cylindrical geometry, constant density (and therefore emissivity) and zero temperature of the electrons. While the first assumption, as any geometrical assumption, is critical for polarization calculations, the second assumption is not very relevant, the important parameter being the total scattering optical depth. The third assumption is apparently very strong - typical maximum temperatures of magnetic CVs are $10-20 \mathrm{keV}$ for polars, and up to $30-40 \mathrm{keV}$ for Intermediate Polars. However, especially for polars and for unpolarized primary emission, the zero temperature approximation is still reasonably good as far as polarization properties 
(see e.g. Poutanen \& Vilhu 1993) and intensity distributions are concerned.

We calculated the polarization properties as a function of two parameters: the radial Thomson optical depth $\left(\tau_{\mathrm{T}}\right)$, and the $H / r_{\mathrm{C}}$ ratio, where $r_{\mathrm{C}}$ is the radius and $H$ the height of the cylinder. Simple modeling of the accretion column gives, for these two parameters, the following equations (e.g. Frank et al. 1993):

$H / r_{\mathrm{C}} \simeq 4.5 \dot{M}_{16}^{-1} f_{-2}^{\frac{1}{2}} M_{\mathrm{wd}, 1}^{\frac{3}{2}} R_{\mathrm{wd}, 9}^{-\frac{1}{2}}$

$\tau_{\mathrm{T}} \simeq 0.08 \dot{M}_{16} f_{-2}^{-1} M_{\mathrm{wd}, 1}^{-\frac{1}{2}} R_{\mathrm{wd}, 9}^{-\frac{1}{2}}$

where $\dot{M}_{16}$ is the accretion rate in units of $10^{16} \mathrm{~g} \mathrm{~s}^{-1}, f_{-2}$ is the fractional area of the column on the White Dwarf surface in units of $10^{-2}\left(f=r_{\mathrm{c}}^{2} / 4 R_{\mathrm{wd}}^{2}\right), M_{\mathrm{wd}, 1}$ the mass of the White Dwarf in units of the solar mass, and $R_{\mathrm{wd}, 9}$ the radius of the White Dwarf in units of $10^{9} \mathrm{~cm}$. $\tau_{\mathrm{T}}$ increases with the accretion rate, and therefore we expect a larger polarization degree when the sources are in their high states. It must also be recalled that Eq. (1) is valid for bremsstrahlung cooling only. If cyclotron cooling is not negligible, Eq. (1) gives only an upper limit to $H / r_{\mathrm{C}}$ (Frank et al. 1993).

To decide the range of parameters to be explored, we assumed $R_{\mathrm{wd}, 9}=1$ and $M_{\mathrm{wd}, 1}=1$, and took the results on the accretion rate and column radius given in Cropper et al. (1998). These parameters were obtained by fitting GINGA spectra with a more complex model, and therefore our procedure is not fully self-consistent, but certainly good enough for an orderof-magnitude estimate.

$\tau_{\mathrm{T}}$ is typically in the range $0.05-1$. This is therefore the range we will explore (for $\tau_{\mathrm{T}} \lesssim 0.05$ the polarization degree is negligible).

$H / r_{\mathrm{C}}$ is typically in the range $1-10$ (apart for sources in low states, when it can became much larger but the optical depth is very low). Given that $H / r_{\mathrm{C}}$ is an upper limit, we extend the explored range down to 0.1 . For $H / r_{\mathrm{C}}$ larger than 10 and smaller than 0.1 , the polarization properties will not change much because the geometry "saturates", apart from very small (in the former case) or large (in the latter case) inclination angles when border effects are still present.

The two parameters are not independent of each other, but $H / r_{\mathrm{C}}$ increases with $f$ and decreases with $\dot{M}$, while $\tau_{\mathrm{T}}$ has the opposite behaviour. We therefore expect large radial optical depths for flattened geometrical configurations. For the sake of completeness, however, we will explore the full parameter space.

1 billion input photons are used for each simulation. The emerging photons are stored in angular bins, with $\Delta \mu=0.1$ ( $\mu=\cos \theta$, where $\theta$ is the inclination angle measured from the cylinder axis, i.e. the angle bewteen the magnetic axis and the line-of-sight).

\section{Results}

The hard (i.e. above $\sim 1 \mathrm{keV}$ ) X-ray emission of magnetic CVs consists not only of plasma (bremsstrahlung) emission but also of a reflection component, arising from the illumination of the
WD surface (e.g. van Teeseling et al. 1994; Beardmore et al. 1995; Matt 1999, and references therein).

We first present the polarization properties of the accretion column alone, and afterwards we will discuss the contribution of the reflection component.

\subsection{Emission from the accretion column}

As long as the scattering matrix does not differ too much from the Rayleigh one, the polarization properties of the X-ray radiation from the accretion column are energy-independent. For obvious symmetry reasons, the polarization is expected to be either parallel or perpendicular to the projection of the cylinder axis onto the plane of the sky. In the former case, as customary when dealing with axisymmetric geometries, we will conventionally assume that the polarization degree, $P$, is negative, while positive of course in the latter case.

In Figs. 1 and 2 , the polarization degree as a function of $\mu$ for different values of $\tau_{\mathrm{T}}$ and $H / r_{\mathrm{C}}$, is shown. For $H / r_{\mathrm{C}} \lesssim 1$, the polarization is negative, as expected for a disc-like geometry and $\tau_{\mathrm{T}}$ less than a few (e.g. Sunyaev \& Titarchuk 1985). Of course, $P$ increases (in absolute value) with $\tau_{\mathrm{T}}$, as more and more photons are scattered (and therefore polarized) before escaping. $P$ at first decreases with increasing $H / r_{\mathrm{C}}$, becoming very small when this parameter is equal to 1 , not suprisingly as in this case the geometry is rather symmetric. Further increasing $H / r_{\mathrm{C}}$, the polarization degree (now positive) starts to increase again.

In Figs. 3 and 4, the flux (in arbitrary units) as a function of $\mu$ is shown. The flux anisotropy increases with increasing $\tau_{\mathrm{C}}$.

The X-ray polarization is expected to be phase-dependent, because of the dependence on the spin phase of $\theta$, which is the angle between the line of sight and the magnetic dipole axis. $\theta$ can be larger than $90^{\circ}$, in which case the accretion column is partly occulted by the WD surface. The degree of polarization in this case depends on the level of occultation, but it is expected to be larger than in the absence of occultation, because the geometry becomes less symmetric.

\subsection{The reflection component}

The reflection component is polarized parallel to the projected cylinder axis (Matt et al. 1989; Matt 1993), i.e. it is negative. The degree of polarization as a function of $\theta$ is shown in Fig. 5, again obtained by means of Monte Carlo simulations (Matt et al. 1989; photons are integrated over the 1-20 keV energy range). The net polarization is then given by:

$P(E, \mu)=\frac{P_{\mathrm{pr}}(\mu)+r(E, \mu) P_{\mathrm{ref}}(E, \mu)}{1+r(E, \mu)}$

where $P_{\mathrm{pr}}$ and $P_{\text {ref }}$ are the polarization degrees of the primary and reflected emission, respectively (taken with their signs), and $r(E, \mu)$ is the ratio between the primary and reflected fluxes (see e.g. Matt 1999). 

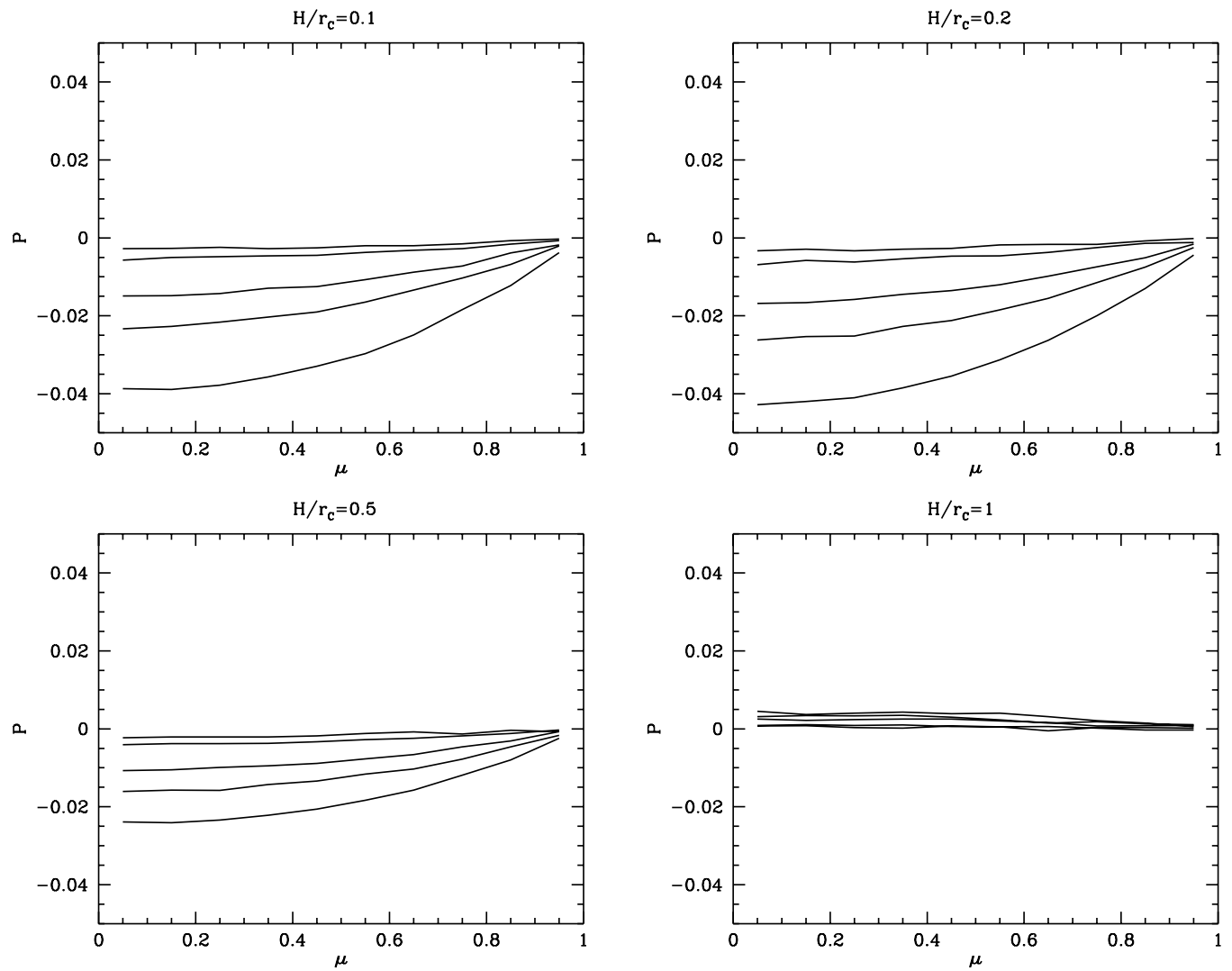

Fig. 1. The degree of polarization, $P$, as a function of $\mu=\cos \theta$ for four values of $H / r_{\mathrm{C}}$. In each panel, five values of $\tau_{\mathrm{T}}$ are shown: 0.05 , 0.1 , $0.3,0.5$ and 1 (in increasing order of the absolute value of $P$ ).
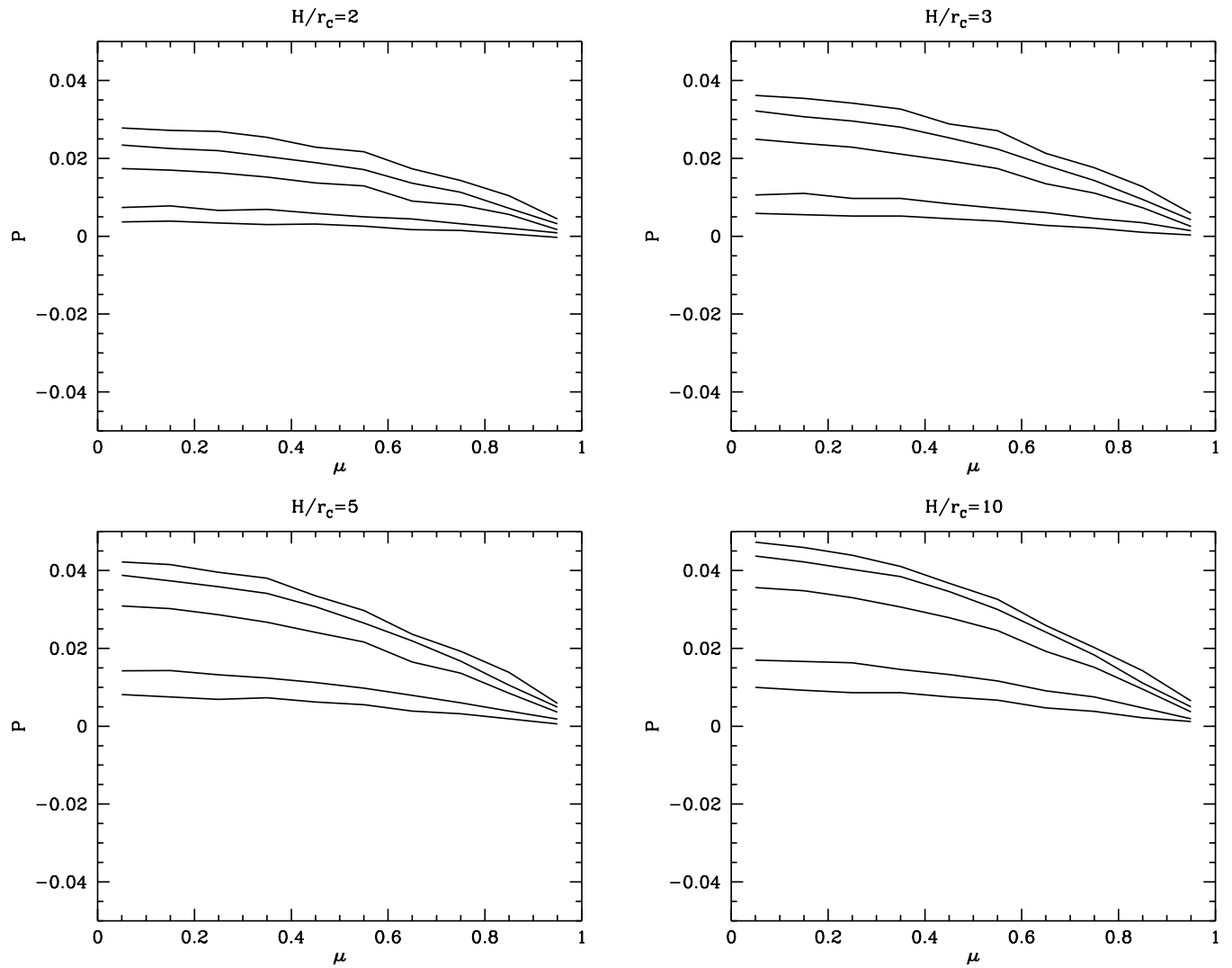

Fig. 2. The same as in the previous figure, for another four values of $H / r_{\mathrm{C}}$. 

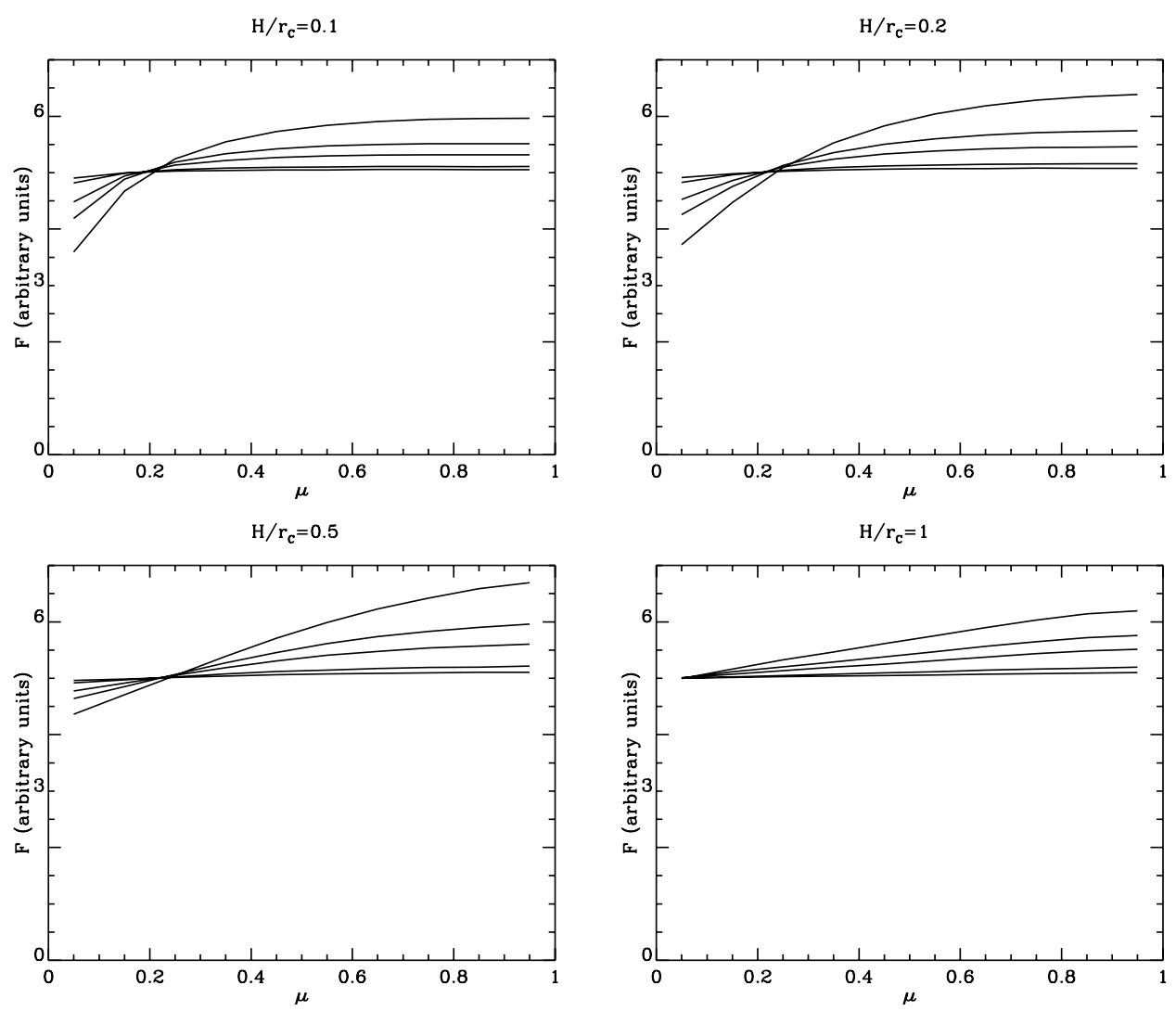

Fig. 3. The flux (in arbitrary units) as a function of $\mu=\cos \theta$ for four values of $H / r_{\mathrm{C}}$. In each panel, five values of $\tau_{\mathrm{T}}$ are shown: $0.05,0.1,0.3$, 0.5 and 1 (in increasing order of anisotropy).
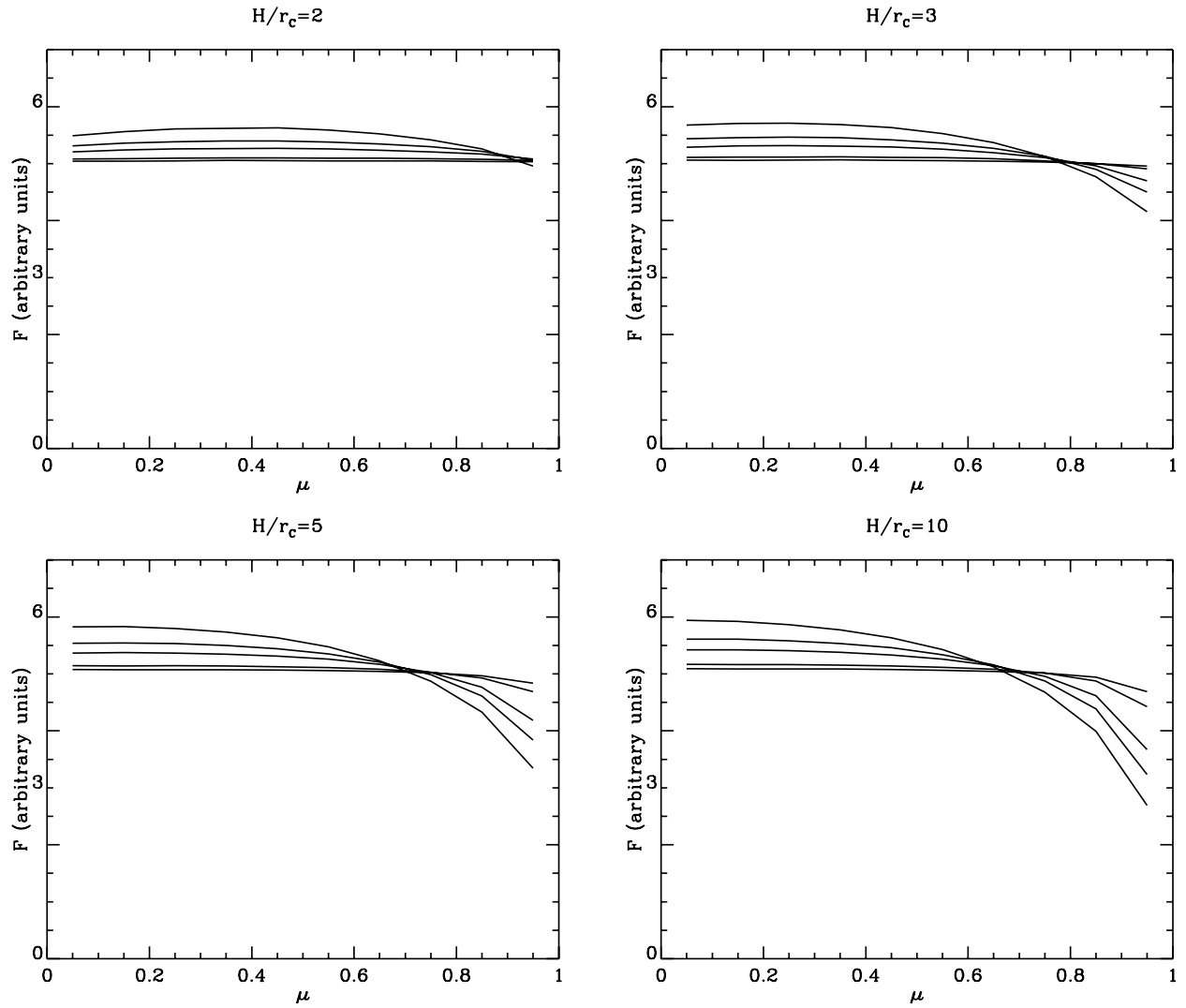

Fig. 4. The same as in the previous figure, for another four values of $H / r_{\mathrm{C}}$. 


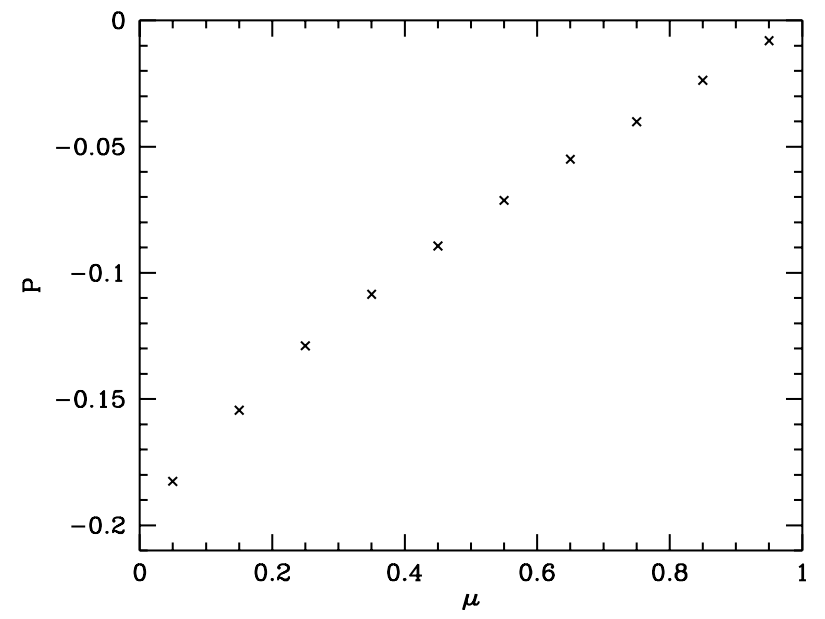

Fig. 5. The polarization degree of the reflected component, as a function of $\mu$.

\subsection{The expected X-ray polarization of AM Herculis}

As an application of our calculations, we discuss the expected phase-dependent X-ray polarization of the archetypal polar, AM Herculis. Assuming the system parameters given by Cropper (1988), i.e. $\beta=61^{\circ}$ and $i=30^{\circ}$ (where $\beta$ is the angle between the magnetic and spin axes, and $i$ the angle between the spin axis and the line-of-sight), $\theta$ never exceeds $90^{\circ}$ significantly, so our calculations can be safely applied to the whole spin period.

The intensity and degree of polarization of the accretion column on the main magnetic pole, without the reflection component (and therefore valid for energies less than a few $\mathrm{keV}$ ), as a function of the spin phase, $\phi$, are shown in Figs. 6 and 7. In Fig. $6, H / r_{\mathrm{C}}=10$ and $\tau_{\mathrm{T}}=0.05$ have been assumed, according to the value derived from the Cropper et al. (1998) estimates from ASCA data. However, during the ASCA observation the source was in an intermediate $\mathrm{X}$-ray state; greater optical depths, and lower $H / r_{\mathrm{C}}$, are expected at higher luminosities. Moreover, the $H / r_{\mathrm{C}}$ values estimated from Eq. (1) are upper limits, as cyclotron cooling is not taken into account. We therefore calculated the phase-dependent polarization properties also for the (more favourable) case of $H / r_{\mathrm{C}}=0.5$ and $\tau_{\mathrm{T}}=0.5$ (Fig. 7).

In the first case (Fig. 6), the polarization degree is low (less than $1 \%$ ) because of the low optical depth. The flux is basically phase-independent, different from what is found in intermediate states by BeppoSAX and ASCA (Ishida et al. 1997; Matt et al. 2000). Unless the real optical depth is larger than derived from Eq. (2), this means that either the assumed geometrical and physical parameters of the accreting column are oversimplified (we have assumed a single temperature and density along the column), or that there is substantial phase-dependent absorption. Studying deviations of the measured polarization from the expected one may help distinguish between the different hypotheses.

The phase-dependent flux shown in Fig. 7 is instead roughly similar to that observed by BeppoSAX during a high state of the source (Matt et al. 2000); the observed amplitude of the modulation is however larger then predicted, again

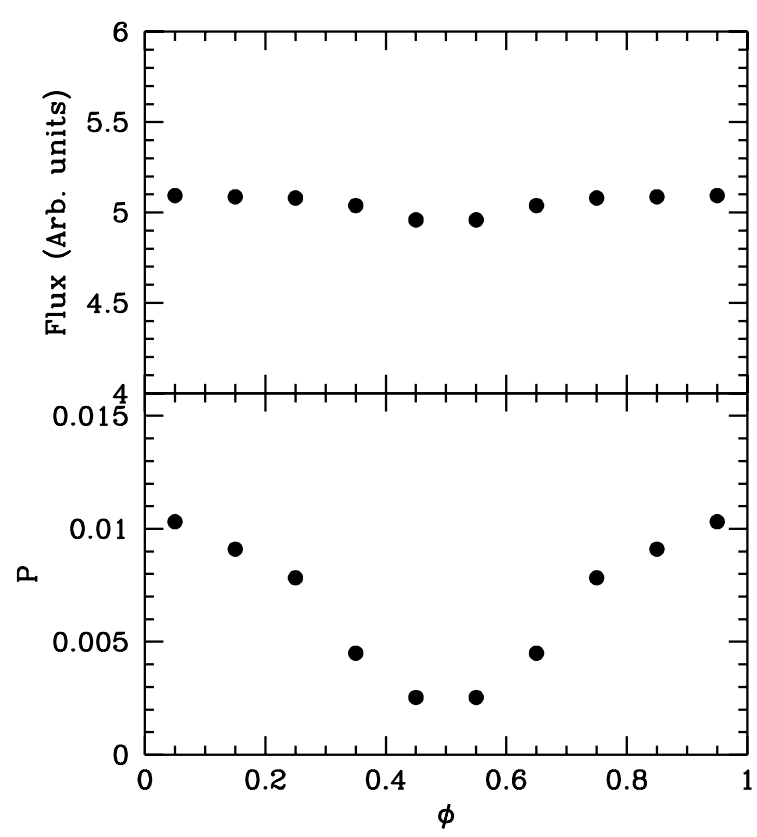

Fig. 6. The intensity (upper panel) and degree of polarization (lower panel) expected for AM Herculis, calculated without the reflection component, as a function of the spin phase. $H / r_{\mathrm{C}}=10$ and $\tau_{\mathrm{T}}=0.05$.

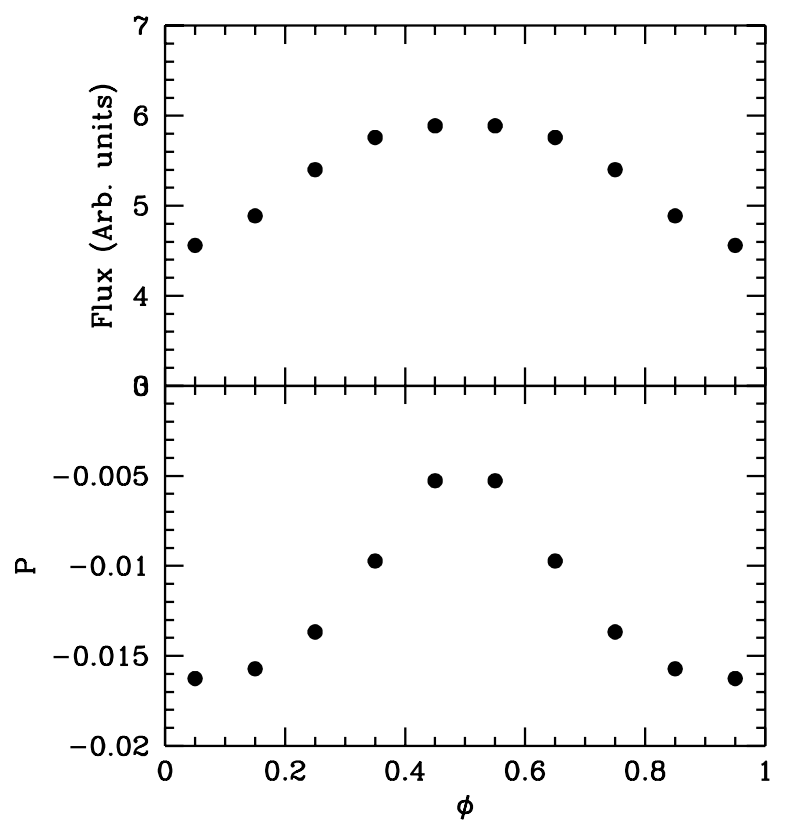

Fig. 7. The intensity (upper panel) and degree of polarization (lower panel) expected for AM Herculis, calculated without the reflection component, as a function of the spin phase. $H / r_{\mathrm{C}}=0.5$ and $\tau_{\mathrm{T}}=0.5$.

suggesting a more complex geometrical and/or physical situation than assumed in our calculations. The polarization degree is expected to be as large as $1.5 \%$, increasing when the flux decreases.

The phase-dependent degree of polarization, including the reflection component as estimated by Matt et al. (2000) during a high state of the source, is shown in Fig. 8 for two energy bins $(5-10 \mathrm{keV}$ and $10-15 \mathrm{keV}) ; R_{\mathrm{C}} / H=10$ and $\tau_{\mathrm{T}}=0.05$ (upper panel) and $R_{\mathrm{C}} / H=0.5$ and $\tau_{\mathrm{T}}=0.5$ (lower panel). 

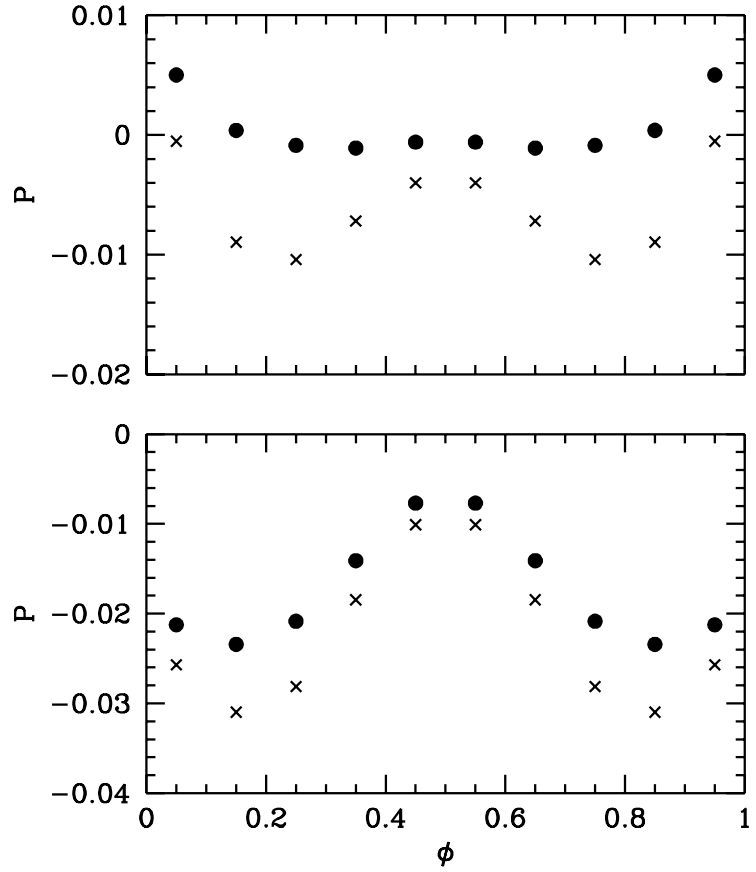

Fig. 8. The degree of polarization, including the reflection component, expected for AM Herculis as a function of the spin phase, for two energy bins (5-10 keV, filled circles, and 10-15 keV, crosses). Upper panel: $H / r_{\mathrm{C}}=10$ and $\tau_{\mathrm{T}}=0.05$; lower panel: $H / r_{\mathrm{C}}=0.5$ and $\tau_{\mathrm{T}}=0.5$.

\section{Summary}

We have calculated the polarization properties of the accretion column in magnetic CVs. Polarization arises because a fraction of the thermal radiation can be Thomson scattered before escaping the column. The polarization degree can be as high as $\sim 4 \%$, and depends on the angle between the magnetic field and the line-of-sight (which varies with the spin phase), which is often well known (e.g. Cropper 1988). It depends also on the Thomson optical depth, $\tau_{\mathrm{T}}$, and on the ratio between the radius and height of the accretion column. If one of these two parameters can be independently estimated (e.g. $\tau_{\mathrm{T}}$ from the iron line broadening, e.g. Hellier et al. 1998), than the other can in principle be deduced by polarization measurements. In particular, as the polarization degree is negative (positive) for $H / r_{\mathrm{C}}$ less (greater) than one, while the polarization of the reflection component (whose importance increases with energy) is always negative, an increase (decrease) of the polarization degree with energy is expected in the former (latter) case.

In this paper we have discussed the continuum emission. However, iron lines provide a significant fraction of the total $\mathrm{X}$-ray flux. Recombination lines emitted in the accretion column may suffer not only Compton scattering but also resonant scattering, so the effective optical depth in the line is larger than in the continuum. Line photons are therefore likely to be more polarized than continuum photons. On the contrary, the fluorescent neutral line emitted by the White Dwarf surface is unpolarized, at least in the line core.

Degrees of polarization of the order of one percent are within the detection capabilities of the new generation X-ray polarimeters based on the photoelectric effect (Costa et al. 2001) when coupled with large enough $X$-ray telescopes (Costa et al. 2003), at least for the brightest magnetic CVs in high state (when their flux can be as high as several millicrabs; for instance the 2-10 keV flux of AM Herculis in high state is about $10^{-10} \mathrm{erg} \mathrm{cm}^{-2} \mathrm{~s}^{-1}$, Matt et al. 2000). Moreover, the spectral resolution should be good enough to search for different polarization degrees in the emission iron lines. Bright magnetic CVs should therefore be added to the traditional lists of targets for future polarimetric missions.

Acknowledgements. I thank Domitilla de Martino and Koji Mukai for very useful comments and suggestions. Financial support from ASI is acknowledged.

\section{References}

Beardmore, A. P., Done, C., Osborne, J. P., \& Ishida, M. 1995, MNRAS, 272, 749

Costa, E., Soffitta, P., Bellazzini, R., et al. 2001, Nature, 411, 662

Costa, E., Soffitta, P., Di Persio, G., et al. 2003, MPE Rep., 281, 235

Cropper, M. 1988, MNRAS, 231, 597

Cropper, M., Ramsay, G., \& Wu, K. 1998, MNRAS, 293, 222

Cropper, M., Wu, K., Ramsay, G., \& Kocabiyik, A. 1999, MNRAS, 306,684

Frank, J., King, A., \& Raine, D. 1992, Accretion power in astrophysics (Cambridge: Cambridge University Press)

Hellier, C., Mukai, K., \& Osborbe, J. P. 1998, MNRAS, 297, 526

Ishida, M., Matsuzaki, K., Fujimoto, R., Mukai, K., \& Osborne, J. P. 1997, MNRAS, 287, 651

McMaster, W. H. 1961, Rev. Mod. Phys., 33, 8

Matt, G. 1993, MNRAS, 260, 663

Matt, G. 1999, Annapolis workshop on magnetic cataclysmic variables, ed. C. Hellier, \& K. Mukai, ASP Conf. Ser., 157, 299

Matt, G., Costa, E., Perola, G. C., \& Piro, L. 1989, in Proc. of the 23rd ESLAB Symposium on two topics in X-ray Astronomy, ed. J. Hunt, \& B. Battrick, ESA SP-296, 991

Matt, G., Feroci, M., Rapisarda, M., \& Costa, E. 1996, Rad. Phys. Chem., 48/4, 403

Matt, G., de Martino, D., Gänsicke, B. T., et al. 2000, A\&A, 358, 177

Poutanen, J., \& Vilhu, O. 1993, A\&A, 275, 337

Sunyaev, R. A., \& Titarchuk, L. G. 1985, A\&A, 143, 374

van Teeseling, A., Heise, J., \& Paerels, F. 1994, A\&A 281, 119

Warner, B. 1995, Cataclysmic variable stars (Cambridge: Cambridge University Press) 